\title{
GROUPS WITH FINITE DIMENSIONAL IRREDUCIBLE REPRESENTATIONS $\left(^{(}\right)$
}

\author{
BY \\ CALVIN C. MOORE $\left({ }^{2}\right)$
}

\begin{abstract}
It will be shown that a locally compact group has a finite bound for the dimensions of its irreducible unitary representations if and only if it has a closed abelian subgroup of finite index. It will further be shown that a locally compact group has all of its irreducible representations of finite dimension if and only if it is a projective limit of Lie groups with the same property, and finally that a Lie group has this property if and only if it has a closed subgroup $\boldsymbol{H}$ of finite index such that $\boldsymbol{H}$ modulo its center is compact.
\end{abstract}

1. Let $G$ be a locally compact group; a (unitary) representation of $G$ is by definition a strongly continuous homomorphism $\pi$ of $G$ into the group of unitary operators on some Hilbert space $H(\pi)$ [11]. One says that $\pi$ is irreducible if the only closed subspaces of $H(\pi)$ invariant under all the operators $\pi(g), g \in G$, are (0) and $H(\pi)$. We shall denote by $\hat{G}$ the set of equivalence classes under unitary equivalence of irreducible unitary representations [11], and we shall use $\pi$ to denote both a representation and its equivalence class. Associated with any representation $\pi$ we have a cardinal number $d(\pi)$, the degree of $\pi$, which is by definition the cardinality of an orthonormal basis of the Hilbert space $H(\pi)$. It is not assumed here that the topology of $G$ satisfies the second axiom of countability nor that $d(\pi)$ is restricted to be $\leqq \boldsymbol{\aleph}_{0}$.

Although, in general, examples show that representations with $d(\pi)$ finite are rather rare, the purpose of this paper is to investigate two closely related hypotheses involving finiteness conditions on $d(\pi)$. To be precise, we say that $G$ satisfies (1) if there is an integer $M<\infty$ such that $d(\pi) \leqq M$ for every $\pi \in \hat{G}$. We say that $G$ satisfies (2) if $d(\pi)<\infty$ for every $\pi \in \hat{G}$. Our results, which are stated below, supply simple necessary and sufficient conditions in terms of the structure of $G$ that a group satisfy either (1) or (2). These questions have been investigated previously and many results obtained. In particular, Freudenthal [3] in 1936 showed that a separable (i.e. second countable) connected group $G$ satisfies (2) if and only if $G=K+V$ where $K$ is compact and $V$ is a vector group. In fact, and this shall be important for us, Freudenthal showed that $G=K+V$ under the much weaker assumption that $G$ is maximally almost periodic; that is, that there exist enough

Received by the editors June 28, 1971.

AMS 1970 subject classifications. Primary 22D05, 22D10, $22 \mathrm{E} 45$.

( $\left.{ }^{1}\right)$ Research supported in part by NSF Grant No. GP-12997.

(2) Sloan Foundation Fellow. 
finite dimensional unitary representations to separate the points of $G$, or equivalently that there is a continuous injection of $G$ into a compact group. Weil [24] extended this result to arbitrary locally compact connected groups. Finally recent work of L. Robertson [20] expands and amplifies our results concerning condition (2). Kaplansky [10] has considered groups $G$ which satisfy condition (1) and proved many interesting results. Condition (1) has also been treated in recent literature (see e.g. [7]) in the context of abstract groups and arbitrary (nontopological) representations.

Finally we mention the results of Thoma ([21], [22]) which completely answer questions about conditions (1) and (2) for discrete groups. Recall that a group is type I [11] if for every unitary representation $\pi$ of $G$, the von Neumann algebra $R(\pi)$ generated by the operators $\pi(g), g \in G$, is type I. Thoma proves that a discrete group is type $\mathrm{I}$ if and only if it possesses an abelian subgroup $A$ of finite index. We note that the existence of such an $A$ implies that $G$ satisfies condition (1) and hence (2) above and that (2) implies that $G$ is type I [9]. Thus conditions (1), (2), type I, and also CCR [11] and existence of an abelian subgroup of finite index are equivalent in the case of discrete groups. This result supplies the key to the proof of Theorem 1 below.

With this introduction, we shall now state our results.

THEOREM 1. If $G$ is locally compact, then there is an integer $M$ such that $d(\pi) \leqq M$ $<\infty$ for all $\pi \in \hat{G}$ if and only if there is an open abelian subgroup of finite index in $G$.

The theorems involving condition (2) involve two definitions. Following [4], we say that $G$ is a $Z$-group (or is a central group) if $G / Z(G)$ is compact where $Z(G)$ is the center of $G$. It is known ([14], [4], [5]) that a $Z$-group satisfies (2), namely that all of its irreducible unitary representations are finite dimensional. Finally we say that $G=\operatorname{proj} \lim \left(G_{\alpha}\right)$ (projective limit) if there is a family of normal subgroups $H_{\alpha}$ directed by inclusion such that $G_{\alpha}=G / H_{\alpha}$ and $\bigcap_{\alpha} H_{\alpha}=(e)$ and such that each $H_{\alpha}$ is compact (or what is really the same, that a cofinal set of the $H_{\alpha}$ is compact).

THEOREM 2. Let $G$ be a Lie group; then $G$ satisfies (2) (i.e. $d(\pi)<\infty \forall \pi \in \hat{G}$ ) if and only if there is an open subgroup $H$ of finite index in $G$ which is a Z-group.

Just to standardize terminology, we say that $G$ is a Lie group by definition if its component of the identity $G_{0}$ is open in $G$, and if $G_{0}$ is a (connected) Lie group. This is equivalent of course to saying that $G$ is locally Euclidean [15].

THEOREM 3. If $G$ is locally compact, then $G$ satisfies (2) if and only if $G=\operatorname{proj} \lim G_{\alpha}$ where each $G_{\alpha}$ is a Lie group which satisfies (2) and thus, equivalently, the structural condition of Theorem 2.

It would be pleasant indeed if Theorem 2, which makes perfect sense for any group $G$, were valid in general. This is unfortunately not the case, and we shall give a simple counterexample in $\$ 5$. 
One may regard the content of Theorems 1 and 2 as saying that the only groups satisfying the relevant conditions on their representations are the obvious ones. In fact the sufficiency of the structural conditions is rather trivial. Theorem 3 is less satisfactory, although it does present necessary and sufficient conditions, and in fact the proof of sufficiency here lies a bit deeper.

2. In this section we want to consider the "if" parts of Theorems 1, 2, and 3 . We mention first the following well-known fact.

Proposition 2.1. Let $G$ be locally compact, and $H$ an open subgroup of finite index. Then $d(\pi) \leqq M<\infty \forall \pi \in \hat{G}$ (resp. $d(\pi)<\infty \forall \pi \in \hat{G}$ ) if and only if $d(\pi) \leqq M^{1}$ $<\infty \forall \pi \in \hat{H}(\operatorname{resp} . d(\pi)<\infty \forall \pi \in \hat{H})$.

Proof. If $H_{0}=\bigcap x \mathrm{Hx}^{-1}(x \in G), H_{0}$ is open and of finite index. So by using our result twice, it suffices to consider the case when $H$ is normal. If $\pi$ is any unitary representation of $G,\left.\pi\right|_{H}$ will denote its restriction to $H$ and if $\lambda$ is any unitary representation of $H, U^{\lambda}$ will denote the induced representation which can be defined with no trouble in this simple situation. As in [13] or as in [19] one can establish a Frobenius reciprocity law of the form $\operatorname{Hom}_{G}\left(U^{\lambda}, \pi\right) \approx \operatorname{Hom}_{H}\left(\lambda,\left.\pi\right|_{H}\right)$ where Hom denotes the space of bounded intertwining operators.

If $\lambda \in \hat{H}$, let us take $\pi=U^{\lambda}$; then, as is clear, $\left.U^{\lambda}\right|_{H}$ is the sum of $n=[G: H]$ irreducible representations all conjugate to $\lambda$. Therefore $\operatorname{Hom}_{H}\left(\lambda,\left.U^{\lambda}\right|_{H}\right)$ has dimension at most $n$, and so does $\operatorname{Hom}_{G}\left(U^{\lambda}, U^{\lambda}\right)$. This says that $U^{\lambda}$ is a finite direct sum of irreducible representations, in fact of at most $n$ irreducible representations. Thus if $d(\pi) \leqq M \forall \pi \in \hat{G}, d\left(U^{\lambda}\right) \leqq n M$, but on the other hand, $d\left(U^{\lambda}\right)=d(\lambda) n$ and so $d(\lambda) \leqq M \forall \lambda \in \hat{H}$. Similarly if $d(\pi)<\infty$ for all $\pi \in \hat{G}$, it follows immediately that $d(\lambda)<\infty$ for all $\lambda \in \hat{G}$.

Conversely suppose that $d(\lambda)<\infty$ for all $\lambda \in \hat{H}$, and let $\pi \in \hat{G}$. Then if $Z\left(\left.\pi\right|_{H}\right)$ is the center of the von Neumann algebra generated by $\left.\pi\right|_{H}, G / H$ acts as a group of automorphisms of $Z\left(\left.\pi\right|_{H}\right)$. Any operator in $Z\left(\left.\pi\right|_{H}\right)$ invariant under this action is a commuting operator for $\pi$ and hence is a scalar. It is immediately clear, then, that the dimension of $Z\left(\left.\pi\right|_{H}\right)$ is at most $n=[G: H]$. Thus $Z\left(\left.\pi\right|_{H}\right)$ is finite dimensional, and it follows that $\left.\pi\right|_{H}$ is a finite sum of primary representations. Since $H$ is type $I$, $\left.\pi\right|_{H}$ has an irreducible subrepresentation $\lambda$.

We apply the Frobenius reciprocity theorem above to $\lambda$ and $\pi$, and conclude that $\operatorname{Hom}_{G}\left(U^{\lambda}, \pi\right) \neq(0)$. A nontrivial commuting homomorphism is necessarily surjective since $\pi$ is irreducible so that $d(\pi) \leqq d\left(U^{\lambda}\right)=n d(\lambda)<\infty$. Moreover if $d(\lambda) \leqq M<\infty$ for all $\lambda \in \hat{H}, d(\pi) \leqq n M$ for all $\pi \in \hat{G}$. This completes the proof of the lemma.

We can dispose of the "if" parts of Theorems 1 and 2 at this point by noting first that $d(\lambda)=1$ for every $\lambda \in \hat{H}$ if $H$ is abelian. Thus if $G$ has an open abelian subgroup of finite index, then $d(\pi) \leqq M$ for some $M$ and all $\pi \in \hat{G}$. Finally if $H$ is a central group, then $d(\lambda)<\infty$ for all $\lambda \in \hat{H}$ by [5] and hence if $G$ has such an open central subgroup of finite index, then $d(\pi)<\infty$ for all $\pi \in \hat{G}$. 
We turn now to the "if" part of Theorem 3 (on projective limits). The following fact accomplishes this and perhaps has some independent interest.

Proposition 2.2. Let $G=\operatorname{proj} \lim G_{\alpha}$ with $G_{\alpha}=G / H_{\alpha}$ where $H_{\alpha}$ is compact. Let $\pi$ be a primary representation of $G$. Then there exists $\alpha$ such that $\pi\left(H_{\alpha}\right)=(1)$.

Proof. Let $\lambda_{\alpha}$ denote the restriction of $\pi_{\alpha}$ to $H_{\alpha}$. Then as $H_{\alpha}$ is compact $\lambda_{\alpha}$ is the sum of copies of various irreducible representations of $H_{\alpha}, \lambda_{\alpha}=\sum n_{\sigma} \sigma\left(\sigma \in \hat{H}_{\alpha}\right)$ where $n_{\sigma}$ are cardinal numbers. Let $X_{\alpha}^{\sigma}$ denote the subspace of $H(\pi)$ which is the range of the projection onto the summand $n_{\sigma} \sigma$. Now, just as in [14], $G$ acts as a permutation group on $\hat{H}_{\alpha}$ and $\pi(g) \cdot X_{\alpha}^{\sigma}=X_{\alpha}^{g \cdot \sigma}$. Thus if $O$ is an orbit of $G$ on $\hat{H}_{\alpha}$ and if $X_{\alpha}^{O}=\sum X_{\alpha}^{\sigma}(\sigma \in O)$ then $X_{\alpha}^{O}$ is an invariant subspace for $\pi$. Moreover the projection $P_{\alpha}^{\sigma}$ onto $X_{\alpha}^{\sigma}$ and hence the projection $P_{\alpha}^{O}$ onto $X_{\alpha}^{O}$ are in the von Neumann algebra $R(\pi)$ generated by $\pi$. Thus $P_{\alpha}^{O}$ is in the center of $R(\pi)$ and, since $\pi$ is primary, $P_{\alpha}^{O}$ is either zero or one. Thus there is a unique orbit $O$ such that $\lambda_{\alpha}=\sum n_{\sigma} \sigma(\sigma \in O)$, with each $\sigma \in O$ of the form $g \cdot \sigma^{\prime}$ for some fixed $\sigma^{\prime} \in O$. Let $H^{\prime}$ be the kernel of $\sigma^{\prime}$ in $H_{\alpha}$. Then $H_{\alpha} / H^{\prime}$ is a Lie group and since $H_{\alpha}=$ proj $\lim \left(H_{\alpha} / H_{\beta}\right)$, $\beta \geqq \alpha$ it follows from general facts about projective limits and Lie groups that there exists $\beta$ such that $H_{\beta} \subset H^{\prime}$. Thus $\sigma^{\prime}(h)=1$ for $h \in H_{\beta}$, but $H_{\beta}$ is normal in $G$ and $\left(g \cdot \sigma^{\prime}\right)(h)=\sigma^{\prime}\left(g h g^{-1}\right)=1$. We find that $\sigma(h)=1$ for all $h \in H_{\beta}$ and $\sigma \in O$, and finally that $\pi(h)=\lambda_{\alpha}(h)=1$ for $h \in H_{\beta}$. This completes the proof.

The "if" part of Theorem 3 is clear, for if $G=\operatorname{proj} \lim G_{\alpha}$, where each $G_{\alpha}$ has all irreducible representations finite dimensional then, by the proposition above, $G$ has the same property.

REMARK. This proposition tells us that the representation theory of a projective $\operatorname{limit} G=\operatorname{proj} \lim G_{\alpha}$ is determined almost completely by the representation theory of the $G_{\alpha}$ 's. For instance it follows at once that $G$ is type I iff each $G_{\alpha}$ is type I, and that $G$ is CCR iff each $G_{\alpha}$ is CCR. Moreover $\hat{G}=\bigcup_{\alpha} \hat{G}_{\alpha}$, and similarly for the primitive ideal spaces of the associated $C^{*}$-algebras.

3. It will be convenient at this point to take care of the "only if" part of Theorem 1 ; that is, we must show that if $G$ is any locally compact group with $d(\pi) \leqq M<\infty$ for some $M$ and all $\pi \in \hat{G}$, then $G$ has an open abelian subgroup of finite index.

Proposition 3.1. If $d(\pi) \leqq m<\infty \forall \pi \in \hat{G}$, then $G$ has an open abelian subgroup of finite index.

Proof. Let $G_{d}$ denote the underlying group of $G$ (with the discrete topology). Then any unitary representation of $G$ is a priori a unitary representation of $G_{d}$ and hence can be viewed as a representation of the algebra $A\left(G_{d}\right)$ where $A\left(G_{d}\right)$ is the group algebra of the discrete group $G_{d}$. Of course $A\left(G_{d}\right)$ is the algebra of all complex measures on $G$ which are supported on a finite set of points with convolution as multiplication. It is clear that the representations of $A\left(G_{d}\right)$ obtained from the irreducible unitary representations of the topological group $G$ separate the elements of $A\left(G_{d}\right)$. Therefore $A\left(G_{d}\right)$ has a separating family of representations 
all of which have degrees no larger than $m$. The "Godement principle" says now that any suitably irreducible representation of $A\left(G_{d}\right)$ can have degree at most $m$. More precisely, there is a standard polynomial in $2 m$ noncommutative variables $P_{2 m}$ such that $P_{2 m}\left(X_{1} \cdots X_{2 m}\right)=0$ is satisfied identically when the $x_{i}$ are $n \times n$ matrices $n \leqq m$, but such that this fails for $(m+1) \times(m+1)$ matrices [8]. Since $A\left(G_{d}\right)$ has a separating family of representations of degree at most $m$, it follows at once that the identity $P_{2 m}=0$ is satisfied identically in $A\left(G_{d}\right)$.

Now if $\pi$ is any representation of $A\left(G_{d}\right)$ on a Banach space $V$, it is clear that the identity $P_{2 m}=0$ holds in the algebra of operators $\pi\left(A\left(G_{d}\right)\right)$. If $\pi$ is completely irreducible in the sense that $\pi\left(A\left(G_{d}\right)\right)$ is dense in the strong operator topology in the algebra of all bounded operators $L(V)$ on $V$, it follows by standard limit arguments that $P_{2 m}=0$ holds identically in $L(V)$. The only way this can happen is of course that $\operatorname{dim} V=d(\pi) \leqq m$. In particular let $\pi$ be any representation of $A\left(G_{d}\right)$ obtained from an irreducible unitary representation of the discrete group $G_{d}$. It is evident that $\pi$ is completely irreducible and hence $d(\pi) \leqq m$. Therefore the discrete group $G_{d}$ is type I and by Thoma's results it has an abelian subgroup $B$ of finite index. The closure of $B$ in the original topology on $G$, say $A$, is clearly abelian and of finite index and hence open. This completes the proof of Theorem 1.

We should remark that we could also have appealed to the results of Isaacs and Passman in [7] and obtained the same result. Moreover if $G$ were separable, we could then find a countable dense subgroup $D$ of $G$. We could then have replaced $G_{d}$ above by $D$ with the discrete topology and the same argument would work; we would find an abelian group $B$ of finite index in $D$. A simple argument shows again that the closure of $B$ in $G$ is abelian and of finite index in $G$. Thus to prove Theorem 1 for separable $G$, Thoma's result for countable discrete groups would suffice.

At this point Theorem 1 is completely proved and the "if" parts of Theorems 2 and 3 are proved. It remains to prove the more nontrivial parts of Theorems 2 and 3, namely, to prove structure theorems about a group $G$ starting from hypotheses about its unitary representations.

Thus we assume that $G$ is a Lie group and that $d(\pi)<\infty$ for all $\pi \in \hat{G}$. Since we want to show that $G$ has an open central subgroup of finite index, we are clearly free to replace $G$ by any subgroup of finite index. Our hypothesis on $G$ is preserved by Proposition 2.1. Let $G_{0}$ be the connected component of $G$. Since the irreducible unitary representations of $G$ separate points of $G$, and hence of $G_{0}, G_{0}$ is maximally almost periodic. By the classical result of Freudenthal and Weil, $G_{0}=K+V$ where $K$ is compact and $V$ is a vector group.

Since our hypothesis on $G$ inherits to any quotient group, $G / G_{0}$ satisfies the same hypotheses. As we have remarked, the hypothesis that $d(\pi)<\infty$ for all $\pi \in \hat{G}$ implies that $G$ is type I. Thus $G / G_{0}$ is type I and, as $G_{0}$ is open in $G$ ( $G$ is a Lie group), $G / G_{0}$ is discrete. Thus Thoma's theorem implies that $G / G_{0}$ has an abelian subgroup of finite index. We may then simply assume that $G / G_{0}$ is abelian. 
Let $N$ be the derived group of $G_{0}=K+V$ so that $N$ is also the derived group of $K$ and is hence a semisimple Lie group. Since $K$ is normal in $G, N$ is also normal in $G$ and $G$ acts by automorphisms of $N$. Since the group of all automorphisms of $N$ modulo its inner ones is finite there is a subgroup of $G$ of finite index which acts by inner automorphisms of $N$. Thus again we can simply assume that all of $G$ acts by inner automorphisms of $N$.

We consider the quotient group $H=G / N$; it is an extension of the discrete abelian group $G / G_{0} \simeq H / H_{0}$ by $H_{0} \simeq G_{0} / N$ which is an abelian connected Lie group. The group $H / H_{0}$ acts on $H_{0}$ and hence on $\hat{H}_{0}$, the dual group. It follows by a trivial modification of Mackey's theory in [14], taking account of the fact that $H / H_{0}$ need not be countable (the extra difficulties here are negligible), that if there is any orbit of $H / H_{0}$ on $\hat{H}_{0}$ which is infinite, then $H$ has an infinite dimensional irreducible unitary representation. Thus if for $\lambda \in \hat{H}_{0}, H(\lambda)$ is the stability group of $\lambda$, then $H(\lambda)$ is of finite index in $H$. Now since $\hat{H}_{0}$ is the sum of a vector space and a finitely generated discrete group, there is a dense finitely generated subgroup, say with generators $\lambda_{i}, i=1, \ldots, n$. Then $\bigcap_{i=1}^{n} H\left(\lambda_{i}\right)$ is of finite index in $H$ and, if $h$ is in this subgroup, it acts trivially on a dense subgroup of $\hat{H}_{0}$, hence on $\hat{H}_{0}$, and hence on $H_{0}$. Thus we may in addition assume that $H_{0}$ is central in $H$.

It remains to analyze the central group extension $1 \rightarrow H_{0} \rightarrow H \rightarrow H / H_{0} \rightarrow 1$. Since $H / H_{0}$ is discrete we can essentially treat this as an extension of abstract groups. It is described by a cohomology class $\alpha \in H^{2}\left(H / H_{0}, H_{0}\right)$. Now for each $\lambda \in \hat{H}_{0}=\operatorname{Hom}\left(H_{0}, T\right)$ where $T$ is the circle group, let $\lambda_{*}(\alpha)$ be the image of $\alpha$ in $H^{2}\left(H / H_{0}, T\right)$ and let $H(\lambda)$ be the corresponding extension of $H / H_{0}$ by $T$. There is a homomorphism $\phi(\lambda)$ of group extensions of $H$ into $H(\lambda)$ such that $T \cdot \phi(\lambda)(H)$ $=H(\lambda)$. Thus if $\pi$ is any irreducible representation of $H(\lambda), \pi$ is scalar on $T$, and hence $\pi \circ \phi(\lambda)$ is an irreducible representation of $H$. Since all irreducible representations of $H$ are finite dimensional, the same is true of $H(\lambda)$ (see [25], [17]).

Now by a standard argument, it follows from this (and, in fact, from the weaker assumption that there is some irreducible representation of finite degree of $H(\lambda)$ nontrivial on $T$ ) that the cocycle $\alpha(\lambda)$ defining $H(\lambda)$ has finite order, say $m$, in $H^{2}\left(H / H_{0}, T\right)$. Since $T$ is divisible we can find some cocycle representative $a(\lambda)$ for $\alpha(\lambda)$ which takes values in $Z_{m}$, the set of $m$ th roots of 1 in $T$. It follows that there is a subgroup $H_{1}(\lambda)$ of $H(\lambda)$ of the form $1 \rightarrow Z_{m} \rightarrow H_{1}(\lambda) \rightarrow H / H_{0} \rightarrow 1$.

It is clear that any irreducible representation of $H_{1}(\lambda)$ extends trivially to an irreducible representation of $H(\lambda)$. Thus all irreducible representations of $H_{1}(\lambda)$ are finite dimensional and, moreover, $H_{1}(\lambda)$ is a discrete group. Thus by Thoma's theorem, there is an abelian subgroup $A_{1}(\lambda)$ of finite index in $H_{1}(\lambda)$ and, since $Z_{m}$ is central, we may assume that $A_{1}(\lambda) \supset Z_{m}$. Now $A(\lambda)=T \cdot A_{1}(\lambda)$ is an abelian subgroup of finite index in $H(\lambda)$ since $T$ is central in $H(\lambda)$.

Now if $s$ and $t$ are in $H / H_{0}$, we may pick coset representatives $\dot{s}, t$ in $H$ for them. The commutator $[\dot{s}, t]$ depends only on $s$ and $t$ and is an element of $H_{0}$ denoted by $[s, t]$. Now for each $\lambda \in \hat{H}_{0}$, we have seen that there is an abelian subgroup $A(\lambda)$ 
of finite index in $H(\lambda)$. This immediately implies that there is a subgroup $B(\lambda)$ of finite index in $H / H_{0}$ such that $\lambda([s, t])=1$ if $s$ and $t$ are in $B(\lambda)$. As before, we use the fact that there is a dense finitely generated subgroup of $\hat{H}_{0}$ with generators $\lambda_{1}, \ldots, \lambda_{n}$, to see that, if $s, t \in \bigcap_{i=1}^{n} B\left(\lambda_{i}\right)$, then $[s, t]=1$. Thus there is a subgroup of finite index in $H$ which is abelian. Now we note that we can assume that $H$ is abelian.

Let us summarize what we know about our original group $G$ now; $G$ has a normal subgroup $N$ which is a compact connected semisimple Lie group, $H=G / N$ is abelian and $G$ acts on $N$ by inner automorphisms. Let $Z$ be the centralizer of $N$ in $G$; then we know that $G=Z N$ and $Z \cap N$ is finite and $Z / Z \cap N \simeq G / N$ is abelian.

We claim now that every irreducible representation of $Z$ is finite dimensional. Indeed if $\pi \in \hat{Z}, \pi(z)=\lambda(z) \cdot 1$ for $z \in Z \cap N$ since $Z \cap N$ is central in $Z$ where $\lambda \in(Z \cap N)^{\wedge}$. We may find a (finite dimensional) irreducible representation $\rho$ of $N$ with $\rho(n)=\lambda(n) \cdot 1$ for $n \in Z \cap N$. Now on the Hilbert space $H(\rho) \otimes H(\pi)$, define a representation $\mu$ of $G$ by $\mu(g)=\rho(n) \otimes \pi(z)$ when $g=n \cdot z, n \in N, z \in Z$. It is immediate that $\mu$ is in fact a well-defined irreducible representation. Since it is finite dimensional, $\pi$ is finite dimensional. Now by exactly the same arguments we used for $H$ we can show that $Z$ has an abelian subgroup of finite index, which we may assume contains $Z \cap N$. Therefore we may assume that $Z$ is abelian in addition to what we have already.

Finally as $G=N \cdot Z$ with $Z$ abelian and centralizing $N$, it is clear that $Z$ is the center of $G$. Then $G / Z \simeq N / Z$ is compact so that $G$ is central. This proves Theorem 2 .

4. It remains to prove the second half of Theorem 3, namely that if $d(\pi)<\infty$ $\forall \pi \in \hat{G}$, then $G$ is a projective limit of Lie groups, $G=$ proj $\lim G_{\alpha}$. Once we know this, then each $G_{\alpha}$ automatically satisfies the same condition that $G$ does and the result will be proved.

Let us say that a representation $\pi$ of $G$ is finite if the von Neumann algebra $R(\pi)$ generated by $\pi$ is a finite von Neumann algebra [2]. This differs somewhat from Mackey's terminology in [11].

LemMA 4.1. If $G$ is locally compact, then $d(\pi)<\infty \forall \pi \in \hat{G}$ if and only if every representation of $G$ is finite.

Proof. The "if" part is clear for an irreducible representation $\pi$ is finite if and only if $d(\pi)<\infty$. For the converse, we consider the group $C^{*}$-algebra $A$ of $G$. If $I$ is a two sided primitive ideal, then $I$ is the kernel of an irreducible representation $\pi$. Since $d(\pi)=n<\infty, A / I$ has dimension $n^{2}$. Let $F_{n}$ be the set of all primitive ideals $I$ of $A$ such that $A / I$ has dimension less than or equal to $n^{2}$ and let $F$ be the primitive ideal space of $A$. Then by assumption $F=\bigcup_{n} F_{n}$. For each subset $K$ of $F$, the kernel $I(K)$ of $K$ is $\bigcap I(I \in K)$. The closure of $K$ in the kernel-hull topology on $F$ is $\bar{K}=\{J \in F ; J \supset I(K)\}$. Let $P_{2 n}$ be the standard polynomial identity characteristic of the ring of $n \times n$ matrices [8]. Then $P_{2 n}=0$ is satisfied in $A / I$ for each $I \in F_{n}$ and 
hence $P_{2 n}=0$ is satisfied in $A / I\left(F_{n}\right)$. Moreover if $J \in \bar{F}_{n}$, then $P_{2 n}=0$ is satisfied in $A / J$ and hence $J \in F_{n}$. Thus $F_{n}$ is a closed subset of $F$.

Now following Glimm [6], we define for each closed subset $K$ of $F$ and for every representation $\lambda$ of $A, P_{\lambda}(K)$ to be the projection onto $H_{\lambda}(K)=\{x: x \in H(\lambda)$, and $\lambda(a) x=0$ for every $a \in I(K)\}$. According to Theorem 1.9 of [6], $K \rightarrow P_{\lambda}(K)$ extends to a countably additive projection-valued measure on the Borel sets of $F$ with $P_{\lambda}(K)$ in the center of $R(\lambda)$. Since $F=\bigcup_{n} F_{n}, H(\lambda)=\sum\left(H_{\lambda}\left(F_{n}\right)-H_{\lambda}\left(F_{n-1}\right)\right)$. We define a subrepresentation $\lambda_{n}$ of $\lambda$ by restricting $\lambda$ to the invariant subspace $H_{\lambda}\left(F_{n}\right)-H_{\lambda}\left(F_{n-1}\right)$. Then $\lambda=\sum \lambda_{n}$, and $\lambda_{n}(a)=0$ if $a \in I\left(F_{n}\right)$. Since $P_{2 n}=0$ is satisfied in $A / I\left(F_{n}\right)$ as we have noted above, the algebra $\lambda_{n}(A)$ and hence its weak closure $R\left(\lambda_{n}\right)$ satisfy $P_{2 n}=0$. Now any von Neumann algebra satisfying this identity is finite. Moreover $R(\lambda)$ is the direct sum of the $R\left(\lambda_{n}\right)$ and since the direct sum of finite algebras is finite [2], $R(\lambda)$ is finite as desired.

LEMmA 4.2. If $d(\pi)<\infty$ for all $\pi \in \hat{G}$, then $G$ has arbitrarily small invariant neighborhoods of $e$.

Proof. We can always find a representation $\lambda$ of $G$ such that $\lambda$ is a homeomorphism of $G$ onto a closed subgroup of the unitary group $U(R(\lambda))$ of $R(\lambda)$ in the strong operator topology (say, the regular representation). If $R(\lambda)$ is finite, which it is by the previous lemma, it is clear that $U(R(\lambda))$ has arbitrarily small neighborhoods of $e$ since $R(\lambda)$ has enough finite traces. It follows at once that $G$ has the same property.

The following lemma completes the proof of Theorem 3.

LEMMA 4.3. If $G$ has arbitrarily small invariant neighborhoods of $e$, then $G$ is a projective limit of Lie groups.

Proof. For the sake of completeness we include a proof of this fact (see also S. P. Wang [23]). We have to show that given any neighborhood $U$ of the identity in $G$, there is a compact normal subgroup $K$ of $G$ with $K \subset U$ such that $G / K$ is a Lie group. We consider first the case when $G$ is totally disconnected. If $U$ is any neighborhood of the identity, it is known [15, p. 54] that there is a compact open subgroup $L$ of $G$ contained in $U$; moreover by assumption there is an invariant neighborhood $V$ of the identity contained in $L$. We let $K=\bigcap g L g^{-1}(g \in G)$ so that $K$ is a compact normal subgroup of $G$. Since $V=g V g^{-1}$ for all $g \in G$, and since $L \supset V, K \supset \bigcap g V g^{-1}(g \in G)=V$ and so $K$ is open. Then $G / K$ is a discrete group, hence a Lie group.

If $G$ is any locally compact group, let $G_{0}$ denote the connected component of the identity in $G$. Then $G / G_{0}$ is totally disconnected, and since $G$ has arbitrarily small invariant neighborhoods, so does $G / G_{0}$. By the above, we can find a compact open normal subgroup $N$ in $G / G_{0}$. We denote by $H$ the complete inverse image of $N$ in $G$ so that $H$ is normal, open and contains $G_{0}$. Since $H / G_{0}$ is compact, it follows $[15$, p. 175] that $H$ is a limit of Lie groups. Therefore if $U$ is any neighborhood of 
the identity in $G$, we can find a compact subgroup $K_{1}$ of $G$, contained in $H$ and normal in $H$, such that $H / K_{1}$ is a Lie group. Our problem is to replace $K_{1}$ by a subgroup $K$ which is also normal in $G$.

Since $H / K_{1}$ is a Lie group, we can find a neighborhood $W$ of the identity in $H / K_{1}$ which contains no nontrivial subgroups. If $p$ is the projection of $H$ onto $H / K_{1}$ we can find by assumption, and the fact that $H$ is open in $G$, an invariant neighborhood $V$ of the identity in $G$ which is contained in $H$ and with $p(V) \subset W$. Again since $H$ is a limit of Lie groups, we can find a compact normal subgroup $K_{2}$ of $H$ with $K_{2} \subset V$, such that $H / K_{2}$ is a Lie group. If $g \in G, g K_{2} g^{-1}$ is a subgroup of $H$ contained in $g V g^{-1}=V$ and hence $p\left(g K_{2} g^{-1}\right)$ is a subgroup of $H / K_{1}$ contained in $p(V)=W$. By the choice of $W, p\left(g K_{2} g^{-1}\right)$ must consist of one element or, equivalently, $g K_{2} g^{-1} \subset K_{1}$. Now let $K$ be the smallest closed subgroup of $G$ containing every $g K_{2} g^{-1}$ for every $g \in G$. Then $K$ is normal in $G$ and $K \subset K_{1} \subset U$ since $K_{1}$ is a subgroup. Moreover $K \supset K_{2}$ by definition and, since $H / K_{2}$ is a Lie group, so is $H / K$. Finally as $H$ is open in $G$, we see that $G / K$ is a Lie group and this completes the proof.

5. In [20] L. Robertson has improved considerably the criterion characterizing non-Lie groups $G$ for which $d(\pi)<\infty$ for all $\pi \in \hat{G}$. The following example shows that Theorem 2 is false in general even for separable groups. Let $T$ be the circle group and let the integers mod two, $Z_{2}$, act on $T$ by conjugation and let $G_{0}=T+Z_{2}$ be the semidirect product. Then $T$ is a compact open subgroup. Let $T^{\infty}$ be an infinite product of circles and let $Z_{2}^{\infty}$ be the countably infinite direct sum of copies of $Z_{2}$. Then $Z_{2}^{\infty}=\left\{\left(a_{i}\right), a_{i}=0,1\right.$, and 0 except for finitely many $\left.i\right\}$ is a discrete group and acts on $T^{\infty}$ componentwise $(a \cdot t)_{i}=a_{i} \cdot t_{i}$ where $t=\left(t_{i}\right) \in T^{\infty}$. Then let $G=T^{\infty}$ $\cdot Z_{2}^{\infty}$ be the semidirect product. ( $G$ can also be described as the restricted direct product [18] of a countable number of copies of $G_{0}$ relative to the compact open subgroup $T$.)

It can be seen either from [14] or from [18] that $d(\pi)<\infty \forall \pi \in \hat{G}$, and in fact that $d(\pi)$ is always a power of 2 . Finally it is quite easy to see that no subgroup of finite index in $G$ can be a central group, so that this is a counterexample to Theorem 2 for general groups.

\section{REFERENCES}

1. L. Auslander and C. C. Moore, Unitary representations of solvable Lie groups, Mem. Amer. Math. Soc. No. 62 (1966). MR 34 \#7723.

2. J. Dixmier, Les algèbres d'opérateurs dans l'espace hilbertien, Cahiers scientifiques, fasc. 25, Gauthier-Villars, Paris, 1957. MR 20 \#1234.

3. H. Freudenthal, Topologische Gruppen mit genügend vielen fastperiodischen Funktionen, Ann. of Math. (2) 37 (1936), 57-77.

4. S. Grosser and M. Moskowitz, On central topological groups, Trans. Amer. Math. Soc. 127 (1967), 317-340. MR 35 \#292.

5. — Representation theory of central topological groups, Trans. Amer. Math. Soc. 129 (1967), 361-390. MR 37 \#5327. 
6. J. Glimm, Families of induced representations, Pacific J. Math. 12 (1962), 885-911. MR 26 \#3819.

7. I. M. Isaacs and D. S. Passman, Groups with representations of bounded degree, Canad. J. Math. 16 (1964), 299-309. MR 29 \#4811.

8. N. Jacobson, Structure of rings, Amer. Math. Soc. Colloq. Publ., vol. 37, Amer. Math. Soc., Providence, R. I., 1956. MR 18, 373.

9. I. Kaplansky, Group algebras in the large, Tôhoku Math. J. (2) 3 (1951), 249-256. MR 14, 58.

10. - Groups with representations of bounded degree, Canad. J. Math. 1 (1949), 105-112. MR 10, 428.

11. G. W. Mackey, Infinite-dimensional group representations, Bull. Amer. Math. Soc. 69 (1963), 628-686. MR 27 \#3745.

12. - Induced representations of locally compact groups. I, Ann. of Math. (2) 55 (1952), 101-139. MR 13, 434.

13. - Induced representations of locally compact groups. II: The Frobenius reciprocity theorem, Ann. of Math. 58 (1953), 193-221. MR 15, 101.

14. - Unitary representations of group extensions. I, Acta Math. 99 (1958), 265-311. MR 20 \#4789.

15. D. Montgomery and L. Zippin, Topological transformation groups, Interscience, New York, 1955. MR 17, 383.

16. C. C. Moore, Extensions and low dimensional cohomology of locally compact groups. I, Trans. Amer. Math. Soc. 113 (1964), 40-63. MR 30 \#2106.

17. - Extensions and low dimensional cohomology of locally compact groups. II, Trans. Amer. Math. Soc. 113 (1964), 64-86. MR 30 \#2106.

18. - Decomposition of unitary representations defined by discrete subgroups of nilpotent groups, Ann. of Math. (2) 82 (1965), 146-182. MR 31 \#5928.

19. - On the Frobenius reciprocity theorem for locally compact groups, Pacific J. Math. 12 (1962), 359-365. MR 25 \#5134.

20. L. C. Robertson, A note on the structure of Moore groups, Bull. Amer. Math. Soc. 75 (1969), 594-599. MR 39 \#7027.

21. E. Thoma, Uber unitäre Darstellungen abzählbarer diskreter Gruppen, Math. Ann. 153 (1964), 111-138. MR 28 \#3332.

22. - Ein Charakterisierung diskreter Gruppen vom Typ I, Invent. Math. 6 (1968), 190-196. MR 40 \#1540.

23. S. Wang, forthcoming.

24. A. Weil, L'intégration dans les groupes topologiques et ses applications, 2nd ed., Hermann, Paris, 1951.

25. H. Weyl, The theory of groups and quantum mechanics, Dover, New York, 1931.

Department of Mathematics, University of California, Berkeley, California 94720 